\title{
Non-spherical Radiation-Driven Wind Models
}

\author{
Joachim Puls ${ }^{1}$, Peter Petrenz ${ }^{1}$, and Stanley P. Owocki ${ }^{2}$ \\ 1 Universitätssternwarte, Scheinerstraße 1, D-81679 München, Germany \\ 2 Bartol Research Institute of the University of Delaware, Newark, DE 19716, USA
}

\begin{abstract}
The present state of modelling radiatively driven stellar winds from rapidly rotating stars is reviewed. Various processes affecting the actual, still controversial wind structure are highlighted, in particular non-radial line-forces and gravity darkening, and useful scaling relations are provided. The importance of accounting for consistent NLTE line-forces depending both on the actual density structure and radiation field (as function of latitude and radius) is stressed, and some independent test calculations confirming earlier numerical results are reported.
\end{abstract}

\section{Geometry and basic assumptions}

In the following, we consider a spherical co-ordinate system with unit vectors $\left(\mathbf{e}_{\theta}, \mathbf{e}_{\phi}, \mathbf{e}_{r}\right)$, where the polar unit vector $\mathbf{e}_{\theta}$ is directed towards the equator (right-handedness). The co-ordinate $\theta$ denotes the co-latitute, i.e., $\theta=0$ at the pole. Our basic assumption concerns the symmetry of the system, which we require to be axisymmetric. Thus, all derivatives with respect to $\phi$ vanish identically in the equations of continuity and momentum (comprising gravity and radiation forces) and the hydrodynmical variables $\left(\rho, v_{\theta}, v_{\phi}, v_{r}, p\right)$ become a function of $(\theta, r, t)$ only. The plasma is considered as isothermal at temperature $T_{\text {eff }}$, which closes the system ( 5 variables, 5 equations) if the appropriate boundary conditions including stellar rotation are specified.

\section{1-D solution in equatorial plane}

The basic effects of rotation on a radiation driven wind can be studied most simply by considering only particles in the equatorial plane (cf. Friend \& Abbott 1986 and Pauldrach, Puls \& Kudritzki (PPK) 1986). With the assumption of a purely radial line acceleration, the angular momentum remains conserved (only central forces), and the rotational speed is given by $v_{\phi}(r)=v_{\text {rot }}\left(R_{*}\right) R_{*} / r$. Thus, the usual equation of motion is modified by the centrifugal acceleration only, which leads to an effective gravity of $G M(1-$ $\Gamma) / r^{2}\left(1-\Omega^{2} R_{*} / r\right)$, where $\Gamma$ accounts for the Thomson-acceleration and $\Omega$ is the ratio of rotational speed at the surface $v_{\text {rot }}\left(R_{*}\right)$ to the break-up velocity $v_{\text {crit }}=v_{\text {esc }} / \sqrt{2}$, with $v_{\text {esc }}$ the photospheric escape velocity.

Without rotation, the mass-loss rate of radiation driven stellar winds scales as (cf. PPK, Kudritzki et al. 1989, Puls et al. 1996) 


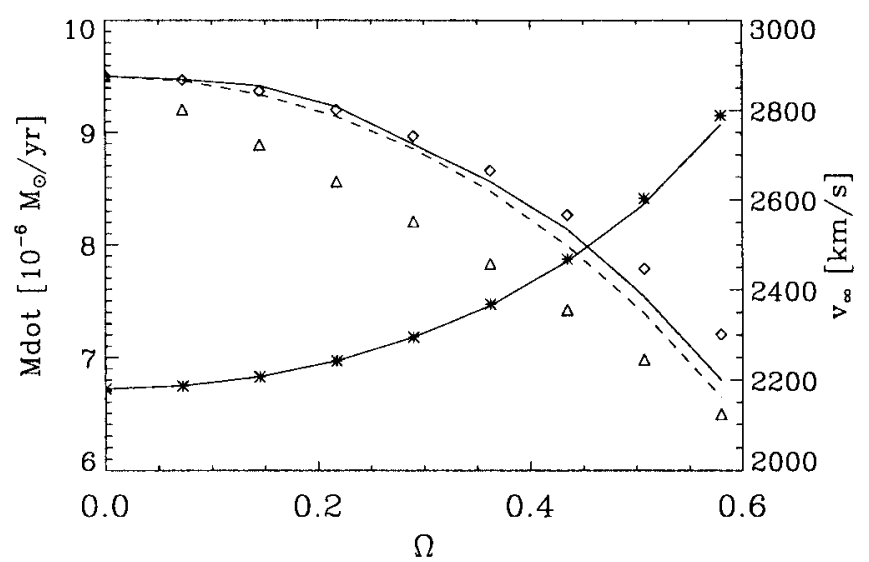

Fig. 1. Influence of rotation on $\dot{M}$ and $v_{\infty}$ for particles in the equatorial plane. Stellar model: "generic OfV star" (cf. PPK) with $v_{\text {crit }}=690 \mathrm{~km} / \mathrm{s}$. Fully drawn: exact solutions; asterisks: scaling relation for $\dot{M}$ (Eq. 2); dashed: new scaling relation for $v_{\infty}$ (Eq. $4, \beta=0.8$ ); diamonds: simple scaling relation for $v_{\infty}$ (Eq. 3); triangles: scaling relation for $v_{\infty}$ by Friend \& Abbott (1986)

$$
\dot{M} \propto(k L)^{1 / \alpha^{\prime}}(M(1-\Gamma))^{1-1 / \alpha^{\prime}}, \alpha^{\prime}=\alpha-\delta
$$

with luminosity $L$ and the usual force-multiplier parameters $(k, \alpha, \delta)$. Since the critical point of the equation of motion (determining $\dot{M}$ ) remains close to the stellar surface if the so-called "finite disk correction factor" is included into the radiation force $(\mathrm{PPK})$, i.e., $R_{*} / r_{\text {crit }} \approx 1$, the only difference induced by rotation is the modification of the effective mass by roughly a factor of $\left(1-\Omega^{2}\right)$. Thus, we expect the mass-loss rate to scale with

$$
\dot{M}(\Omega)=\dot{M}(0)\left(1-\Omega^{2}\right)^{1-1 / \alpha^{\prime}}
$$

A comparison with the "exact" solution obtained from the code developed by PPK confirms the validity of this expression (Fig. 1). Since the scaling law for the terminal velocity (without rotation) suggests a proportionality $v_{\infty} \propto v_{\text {esc }} \propto(M(1-\Gamma))^{1 / 2}$, the most simple idea to include rotation is

$$
v_{\infty}(\Omega)=v_{\infty}(0)\left(1-\Omega^{2}\right)^{\frac{1}{2}}
$$

A slightly more refined investigation accounting for the change of $\dot{M}$ and the decreasing influence of the centrifugal support in the outer wind leads to a more complicated expression, where $x=v_{\infty}(\Omega) / v_{\infty}(0)$ results from the solution of the non-linear equation 


$$
x^{2}=\left(1-\Omega^{2}\right)^{1-\alpha^{\prime}} x^{2 \alpha-\delta}\left(1+\frac{v_{\mathrm{esc}}^{2}}{2 \beta v_{\infty}^{2}(0)}\right)-\frac{v_{\mathrm{esc}}^{2}}{2 \beta v_{\infty}^{2}(0)},
$$

which collapses to Eq. 3 for small values of $v_{\text {esc }} / v_{\infty}(0)$ and $\delta .(\beta \approx 0.7 \ldots 1.3$ is the exponent of the typical velocity law valid for radiation driven winds). A comparison of both relations with the exact solution as well as with the expression provided by Friend \& Abbott (1986) is given in Fig. 1.

\section{Wind compressed disks and zones}

The details of rotationally induced wind compressed disks (WCDs) and wind compressed zones (WCZs) (Bjorkman \& Cassinelli 1993) are reviewed by J. Bjorkman elsewhere in this volume. The basic idea follows again from the assumption of purely radial line forces. Thus, the specific angular momentum is conserved not only for particles in the equatorial plane, but for all particles, and their motion is restricted to the orbital plane to which they belong (tilted by an angle of co-latitude $\theta_{0}$ from which they start). Furthermore, pressure forces are negligible in the supersonic region, and the free flow of the particles can be simulated by a corresponding 1-D treatment as above, however, of course, with a centrifugal acceleration modified for the actual azimuthal velocity in the orbital plane, $v_{\phi}(r)=v_{\phi}(\Theta=\pi / 2, r) \sin \theta_{0}$. Thus, the mass-loss rates and terminal velocities for particles starting at $\theta_{0}$ follow the same scaling relations as above, however with $\Omega^{2}$ replaced by $\left(\Omega \sin \theta_{0}\right)^{2}$ : $\dot{M}$ increases and $v_{\infty}$ decreases towards the equator! In the course of the particles' motion, their azimuthal angle $\Phi^{\prime}(r)$ in the orbital plane is changing, and they are deflected towards the equator. If $\Phi^{\prime}$ becomes larger/equal $\pi / 2$, the particles would cross the equator, where they collide supersonically with particles from the other hemisphere: finally, a wind compressed disk is formed by this process. (For further consequences, $\mathrm{cf}$. Bjorkman, this volume.) If we assume a $\beta$-law for the radial velocity component, $v_{r}(r)=v_{\infty}(1-b / r)^{\beta}$ with $b$ derived from the minimum velocity at $r=R_{*}$, we find for the asymptotic azimuthal angle

$$
\Phi^{\prime}(r \rightarrow \infty)=\frac{1}{\beta-1} \frac{R_{*}}{b} \frac{v_{\text {rot }}\left(R_{*}\right) \sin \theta_{0}}{v_{\infty}\left(\theta_{0}\right)}\left(1-\frac{b}{R_{*}}\right)^{1-\beta},
$$

which immediately suggests that disk formation or a significant compression towards the equator can occur only under the following conditions ( $\Phi^{\prime}$ has to be large): large $v_{\text {rot }}$, small $v_{\infty}$, flat velocity field, i.e., large $\beta$. It is important to realize that models with a typical velocity law $(\beta=0.7 \ldots 0.8)$ give rise to only weak compression factors unless the ratio $v_{\infty} / v_{\text {rot }}$ is extremely low.

Using the same assumptions as outlined above (radial line force), Owocki, Cranmer \& Blondin (1994) confirmed the analytical WCD model by performing time-dependent hydrodynamical simulations. Their results were, except from some interesting details, in good agreement with the predictions by Bjorkman \& Cassinelli. In order to obtain a compressed disk, however, they 
had to use a fairly small value of $\alpha^{\prime}=0.35,(\alpha=0.51, \delta=0.16)$, since for larger values the radial velocity gradient became too large to lead to disk formation, in agreement with the above constraints.

\section{Non-radial line forces}

All results derived so far rely on the assumption of purely radial line-forces, implying the conservation of angular momentum. The line acceleration, however, is a vector quantity, defined per line by (in the Sobolev limit and accounting for an optically thin continuum)

$$
\mathbf{g}^{\mathrm{rad}}(\mathbf{r})=\frac{\bar{\chi}_{L}(\mathbf{r})}{c \rho(\mathbf{r})} \int_{\omega_{\mathrm{c}}} I_{*}(\mathbf{n}) \mathbf{n} \frac{1-\mathrm{e}^{-\tau_{\mathrm{S}}(\mathbf{r}, \mathbf{n})}}{\tau_{\mathrm{S}}(\mathbf{r}, \mathbf{n})} \mathrm{d} \omega ; \tau_{\mathrm{S}}(\mathbf{r}, \mathbf{n})=\frac{\bar{\chi}_{L}(\mathbf{r}) \lambda}{|\mathbf{n} \cdot \boldsymbol{\nabla}(\mathbf{n v}(\mathbf{r}))|}
$$

where $\bar{\chi}_{L}$ is the frequency integrated line-opacity, $\omega_{\mathrm{c}}$ the solid angle subtended by the stellar disk, $I_{*}$ the stellar intensity, $\tau_{\mathcal{S}}$ the Sobolev optical depth and the denominator of $\tau_{\mathrm{S}}$ contains the directional derivative of the local velocity field. From Eq. 6, it is obvious that non-radial components of $\mathbf{g}^{\text {rad }}$ are present under two conditions: either the stellar intensity depends on direction (next section), or $\tau_{\mathrm{S}}$ depends on direction. Note, that in the latter case for $I_{*}=$ const only optically thick lines induce non-radial components, which are then proportional to asymmetries in the derivative. Speaking in physical terms, the resulting accelerations depend on the differences in both intensities and optical depths along the various contributing lines of sight.

Let us first consider the azimuthal component $g_{\phi}$. For this quantity, the directional derivative can be written as $f_{r}+\sin \varphi f\left(\frac{\partial\left(v_{\Phi} / r\right)}{\partial r}\right)$, where $f_{r}$ is the usual symmetric radial component, and $\sin \varphi$ is proportional to the azimuthal component of $\mathbf{n}$ (defined in the same sense as $\Phi$ ). Thus, the direction of the resulting azimuthal acceleration depends only on the sign of $\partial\left(v_{\Phi} / r\right) / \partial r$, which is negative in all cases of rotational laws slower than solid rotation. As a result, even for a symmetric core intensity (i.e., only radial fluxes), optically thick lines induce an acceleration antiparallel to $\mathbf{e}_{\Phi}$, i.e., a spin-down of the wind and loss of angular momentum. This effect was firstly investigated by Grinin (1978). For a further discussion, see Owocki et al. (1997) and Owocki, this volume.

With respect to $g_{\Theta}$, the argumention is analogous. The decisive term in the velocity gradient inducing the required asymmetry is given here by $r \frac{\partial\left(v_{\Theta} / r\right)}{\partial r}+\frac{\partial v_{r}}{r \partial \theta}$, where the second term is the important one. As long as the radial velocity law at the equator is slower than in polar regions - an almost inevitable consequence of rotation due to the reduced escape velocity $\propto\left(1-\Omega^{2} \sin ^{2} \Theta\right)^{1 / 2}-$, this gradient is negative, implying a larger total directional derivative (including the symmetric radial component) towards the pole than towards the equator: Again, $g_{\Theta}$ is anti-parallel to $\mathbf{e}_{\Theta}$ ! Note, 
that this direction could be only reversed if the radial expansion at the pole were slower than at the equator.

Finally, by summing up the contribution of all lines (via folding with the line-strength distribution function), a polewards acceleration is created which is sufficient to stop the equatorwards motion predicted by WCD/WCZmodels and actually reverses its direction. This result (for details see the "stopping length analysis" by Owocki et al. 1997) is the so-called "inhibition effect" (Owocki et al. 1996, Bjorkman, this volume), which inhibits the formation of a wind-compressed disk due to the reversal of the (supersonic) velocities $v_{\Theta}$, which are directed towards the equator only under the assumption of purely radial line-forces.

\section{Distortion of the surface and gravity darkening}

Because of the centrifugal acceleration $\propto \sin \Theta$, the surface of the star becomes distorted. The degree of deformation can be calculated by using an appropriate Roche potential (Collins 1963, Collins \& Harrington 1966). Some applications are given by Cranmer \& Owocki (1995) and Petrenz \& Puls (1996). Moreover, von Zeipel's theorem may be used (for a discussion, see A. Maeder, this volume), which relates the flux and thus the effective temperature with the effective gravity as function of $\Theta$. In consequence, the poles become hotter and the equator cooler than the average $T_{\text {eff }}$ appropriate for the stellar luminosity. This change in the radiation field has a number of consequences: at first, the ionization/excitation balance becomes a function of co-latitude due to the varying radiation field (next section). Second, the polar wind plasma is irradiated by a larger flux compared to the equatorial one, which enhances the radiative acceleration and thus the expected massloss from this region. For a wind with typical exponent $\alpha^{\prime}=0.5$ illuminated by a uniformly bright disk, the mass-flux varies as

$$
\dot{M}(\Theta) \propto\left(1-\Omega^{2} \sin ^{2} \Theta\right)^{-1} \quad \alpha^{\prime}=0.5, \text { uniform disk }
$$

(cf. Eqs. 1, 2), i.e., increases from pole to equator. If one accounts for gravity darkening, however, $L^{1 / \alpha^{\prime}} \rightarrow\left(F(\Theta) R_{*}^{2}(\Theta)\right)^{1 / \alpha^{\prime}} \sim\left(g_{\mathrm{eff}}(\Theta) R_{*}^{2}(\Theta)\right)^{1 / \alpha^{\prime}}$, an additional dependence on $\left[M_{\mathrm{eff}}\left(1-\Omega^{2} \sin ^{2} \Theta\right)\right]^{1 / \alpha^{\prime}}$ is created. In total, we find

$$
\dot{M}(\Theta) \propto\left(1-\Omega^{2} \sin ^{2} \Theta\right)^{+1} \quad \text { with gravity darkening }
$$

(cf. Owocki et al. 1997, 1997a). Thus, the inclusion of gravity darkening (increased polar radiation flux) can reverse the situation and leads to a larger polar mass-flux.

Finally, the absorbing lines at mid-latitudes are irradiated by an asymmetric intensity, where the polar contribution is stronger and hotter than the equatorial one. This effect partially counteracts the optical depth effect 

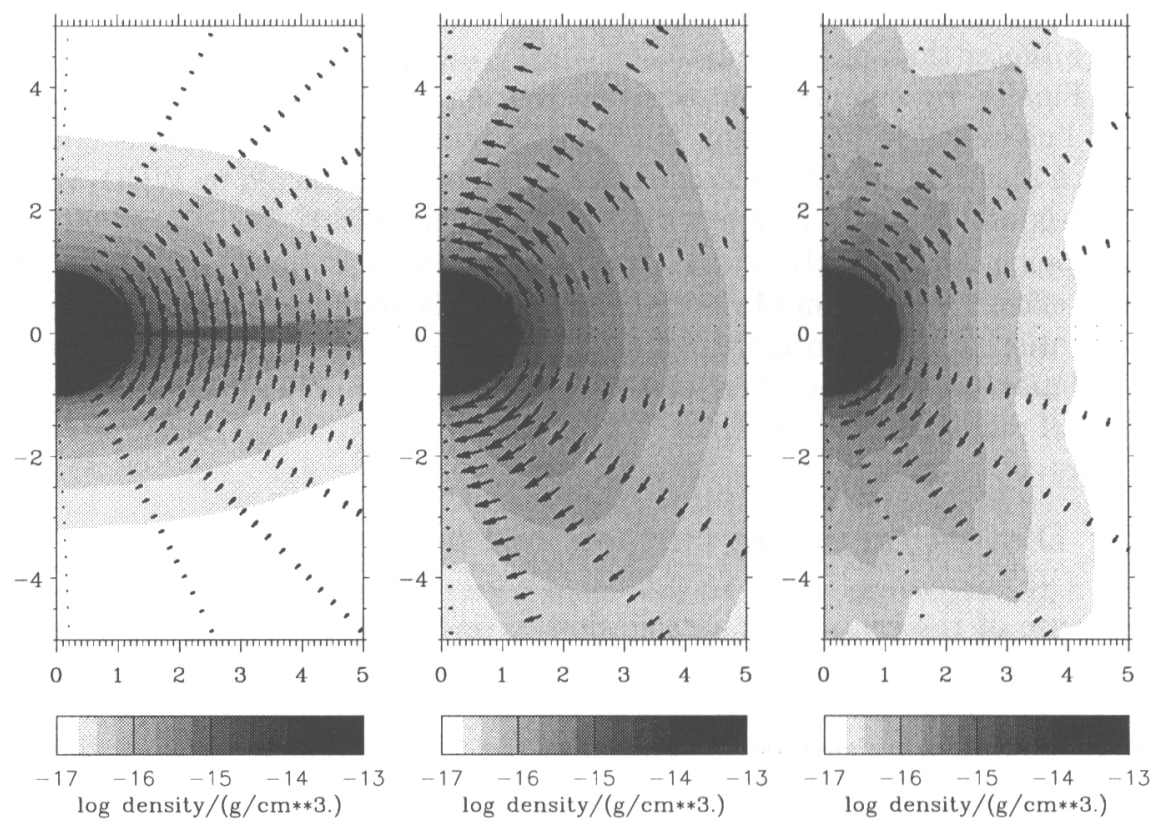

Fig. 2. Radiation driven wind models for a rapidly rotating B-star (see text).

Model paramters (mean values): $T_{\mathrm{eff}}=20,000 \mathrm{~K}, \log g=4.1, R_{*}=4 R_{\odot}$, $v_{\text {esc }}=690 \mathrm{~km} / \mathrm{s}, v_{\text {rot }}=350 \mathrm{~km} / \mathrm{s}$, line force parameters: $k=0.61, \alpha=0.51$, $\delta=0.16 \rightarrow \dot{M}=3 \cdot 10^{-10} M_{\odot} /$ yr.

Hydrodynamics: ZEUS-2D (Stone \& Norman 1992). Lower boundary: rotationally distorted, stair-casing (Owocki et al. 1994) plus "no slip boundary condition".

(cf. Eq. 6) and leads to a meridional acceleration $g_{\Theta}$ being smaller than in the uniformly bright disk case, however still sufficiently large to induce a polewards directed velocity.

As a brief summary and as an independent confirmation of the various numerical models published so far, Fig. 2 shows three different wind models for a rapidly rotating B-star calculated by one of us (P.P.) using the ZEUS2D-code (Stone \& Norman 1992), accounting for rotational distortion of the surface (for details, see caption). The first one (left) displays the formation of a wind-compressed disk, if only radial line-forces are accounted for. The second one (middle) demonstrates the inhibition effect, if non-radial forces are included and a uniformly bright stellar disk is assumed. This model shows the largest mass-flux still at the equator (Eq. 7). Model 3 (right) accounts additionally for gravity darkening: Now, the mass-flux is highest at the poles (Eq. 8), and the polar velocities (arrows, on scale) are smaller than for Model 2 , in agreement with our reasoning from above. 


\section{Outlook - Consistent NLTE line forces}

In all calculations performed so far, the total line force was calculated within the force-multiplier concept, where the corresponding parameters were assumed to be constant throughout the wind, i.e., independent both of $r$ and $\Theta$ and taken from tables designed to cope with the 1-D situation. One has to remember, however, that these parameters are (or more precisely: should be) the result of consistent NLTE-calculations, which are affected by at least two processes: i) The $\Theta$-dependence of both density and illuminating radiation field can have a significant effect on the ionization stratification, which in turn leads to a variation of the force-multiplier parameters with respect to co-latitude. As an example, Fig. 3 displays the ionizing radiation field for the rapidly rotating $B$-star wind model discussed above, when gravity darkening is included. The differences with respect to $\Theta$ are largest $(\approx 5000 \mathrm{~K})$ close to the surface, where the ionization is controlled by the local radiation field $\propto T_{\text {eff }}(\Theta)$. At larger distances from the surface, the plasma "sees" radiation from all angles between pole and equator, so that the radiation temperatures converge to similar values for $r \rightarrow \infty$. ii) Second, the assumption of constant force-multiplier parameters as function of $r$ is rather questionable even for a uniformly bright disk (cf. Kudritzki et al. 1997). Fig. 4 displays the behaviour of $\alpha$ for a spherical wind in dependence on the optical depth variable $t \propto \rho /(\mathrm{d} v / \mathrm{d} r)$ and different "mean densities" $\log n_{\mathrm{e}} / W$. The overall trend of $\alpha$ becoming smaller for decreasing $t$ reflects the decreasing influence of optically thick lines on the acceleration, if the wind plasma becomes thinner in the course of its outward trajectory. The reader may note, however, that the specific behaviour of $\alpha(t)$ varies strongly with spectral type and mean wind density (i.e., in concert with the dominant ions): one finds also cases with $\alpha$ increasing with decreasing $t$ at first, before this behaviour is finally reversed.

Presently, incorporation of these effects into a consistent 2-D description is under way in our working group. A number of simulations for rapidly rotating $\mathrm{O}$-star winds with significant mass-loss have been performed, to demonstrate operation of the basic algorithms. Before specific results can be published, however, some final test calculations have to be undertaken. Especially in those cases as displayed in Fig. 4, when $\alpha$ is decreasing with $t$, a number of interesting effects are to be expected, since then the acceleration of the outer wind (low $\alpha$ ) can become too small for the mass-loss rate created at the wind base (larger $\alpha$ ).- Hopefully, the final simulations will help to answer the question concerning the "real" wind structure of rapidly rotating winds as function of spectral type and luminosity class. Note, however, that magnetic fields if present with sufficient strength (cf. Mathys, this volume) could again alter the conclusions.

One of our final goals is the incorporation of continuum optical depth effects which in the B-supergiant domain can induce the so-called "bi-stability" mechanism (Pauldrach \& Puls 1990). The decisive quantity which controls this behaviour is the optical depth in the Lyman continuum, which - for 

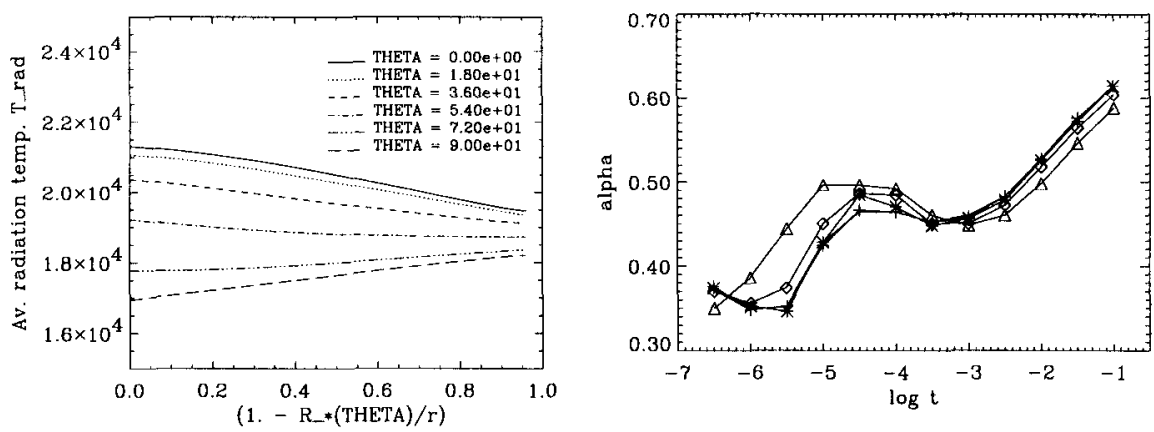

Fig. 3. Ionizing radiation temperature for a rapidly rotating B-star wind model (cf. Fig. 2) including gravity darkening, averaged over illuminating disk, as function of $\Theta$ and height over surface.

Fig. 4. NLTE-force multiplier parameter $\alpha$ as function of optical depth variable $t \propto \rho /(\mathrm{d} v / \mathrm{d} r)$ for a one-dimensional wind with $T_{\text {eff }}=20000 \mathrm{~K}$ and different "densities" $\log n_{\mathrm{e}} / W=11.5$ (asterisks), 11.0, 10.5, 10.0, 9.5 (triangles). Illuminating photospheric spectrum from Kurucz fluxes (Kudritzki \& Springmann, priv. comm.).

rotating stars and accouting for gravity darkening - depends sensitively on rotationally induced variations of wind density and radiation temperature and thus becomes a strongly varying function of $\Theta$ (Lamers \& Pauldrach 1991; Lamers, this volume, however also Owocki et al. 1997). Due to this behaviour, the bi-stability effect is thought to be responsible for the $\mathrm{B}[\mathrm{e}]$ phenomenon (Lamers \& Pauldrach 1991) if the star rotates close to break-up and has a rather large (undisturbed) polar mass-loss. It has to be seen if the observed bimodal B[e] wind structure (e.g. Zickgraf et al., this volume) can be actually explained by simulations accounting for consistent line-forces.

\section{References}

Bjorkman, J.E., Cassinelli, J.P., 1993, ApJ 409, 429

Collins, G.W.II, 1963, ApJ 138, 1134

Collins, G.W.II, Harrington, J.P., 1966, ApJ 146, 152

Cranmer, S.R., Owocki, S.P., 1995, ApJ 440, 308

Friend, D.B., Abbott, D.C., 1986, ApJ 311, 701

Grinin, A., 1978, Sov. Astr. 14, 113

Kudritzki, R.P., Pauldrach, A., Puls, J., Abbott, D.C., 1989, A\&A 219, 205

Kudritzki, R.P., Springmann, U., Puls, J., et al., 1997, PASPC 131, 299

Lamers, H.J.G.L.M., Pauldrach A.W.A., 1991, A\&A 244, L5

Owocki, S.P., Cranmer, S.R., Blondin, J.M., 1994, ApJ 424, 887

Owocki S.P., Cranmer S.R., Gayley, K.G., 1996, ApJ 472, L1150

Owocki, S.P., Cranmer, S.R., Gayley, K.G., 1997. In: Proceedings of Workshop on B[e] Stars, held in Paris, France, June, 1997, ed. A. Hubert, Kluwer, in press. 
Owocki, S.P., Gayley, K.G., Cranmer, S.R., 1997a, PASPC 131, 237

Pauldrach, A.W.A., Puls, J., Kudritzki, R.P., 1986, A\&A 164, 86 (PPK)

Pauldrach, A.W.A., Puls, J., 1990, A\&A 237, 409

Petrenz, P., Puls, J., 1996, A\&A 312, 195

Puls, J., Kudritzki, R.-P., Herrero, A., et. al., 1996, A\&A, 305, 171

Stone, J.M., Norman, M.L., 1992, ApJS 80, 753

\section{Discussion}

N. Langer: The von Zeipel theorem is based on assumptions which do not hold for the luminous stars you consider. It should not be applied to these stars.

J. Puls: We are well aware of this problem. In our present philosophy, however, we regard the temperature difference we obtain from the von Zeipel theorem as a sort of maximum effect which has to be investigated.

A. Maeder: Corrections to the von Zeipel theorem due to the non-cylindrical rotation and the fact that real stars are not barotropic are leading to still higher polar flux; thus this would even reinforce the polar mass flux.

J. Puls: So, we even have to correct our previous maximum temperature difference argument. In this case, the resultant structure would be even more elongated in the polar direction.

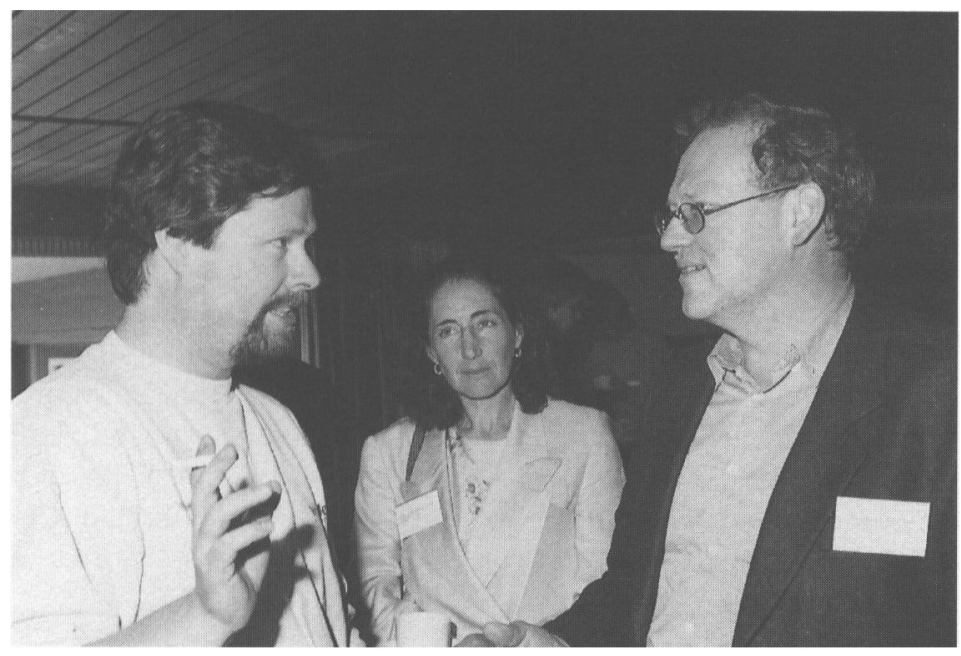

Joachim Puls, Gloria Koenigsberger and Wolfgang Glatzel 